\title{
Novel KIAA1109 variants affecting splicing in a Russian family with ALKURAYA-KUČINSKAS syndrome
}

To the Editor

Despite the application of chromosomal microarray analysis (CMA) and whole exome sequencing (WES), the genetic diagnosis of fetal abnormalities is still established in less than $50 \%$ of the cases. ${ }^{1}$ One of the reasons may be the fact that our knowledge about genes associated with structural disorders is not complete. There are at least several dozen genes that are intolerant to functional variation and lethal in mice, but are not associated with human disease. ${ }^{2}$ It suggests that these genes could be under strong negative selection and mutations in them lead to embryonic/fetal abnormality and death. Thus, we assume that the number of genes implicated in the disorders of early development might be much greater, but they escape our attention due to restricted application of WES in diagnostics of fetal abnormalities.

Here, we report a Russian family with three miscarriages and fetal abnormalities. The family gave informed consent for this study, which was approved by the Institutional Review Board. A 29-year-old female and her 31-year-old husband were asymptomatic and did not have family history of the disease. The couple's first pregnancy (II.1) resulted in a spontaneous miscarriage at 7 to 8 weeks. The second pregnancy (II.2) was terminated at 13 weeks of gestation because of ultrasonographic fetal abnormalities. Cytogenetic testing of parents revealed normal karyotype. During the third pregnancy (II.3), the following ultrasound anomalies were observed at 14.1 weeks: bilateral ventriculomegaly, arthrogryposis (radial clubhand, bilateral clubfoot, flexed deformity of hip, knee and ankle joints), bilateral pyelectasis, increased thickness of the nuchal-fold, hypoplastic and low set ears, a defect of the interventricular septum was suspected (Figure 1C). The family opted for pregnancy termination and genetic analysis.

CMA of the DNA extracted from chorionic villus sampling (CVS) revealed no chromosomal pathology. Subsequent WES of fetal DNA (average ontarget coverage 95.4x) did not identify any disease-causing variants in known genes at the time of the report. Nevertheless, we identified two novel intronic variants in the KIAA1109 gene: NM_015312.3:c.1932-3A>G, NM_015312.3: c. $2613+1 \mathrm{G}>\mathrm{A}$. This gene has not been associated with any hereditary disease, but one loss-of-function variant was reported in homozygous state in a patient with similar clinical manifestations. ${ }^{3}$ Sanger sequencing analysis revealed that the polymalformative fetus had compound heterozygous KIAA1109 variants (Figure 1B).

We performed analysis of KIAA1109 pre-mRNA splicing using reverse transcription polymerase chain reaction of RNA obtained from parents' blood samples. We showed that c.1932-3A>G variant disrupts native splicing, resulting in a two-nucleotide extension of exon 17 (Figure 1E) and leads to a frameshift and truncated protein: NP_056127.2:p.(S645Gfs*32). While c.2613+1G>A variant leads to a skipping of exon 20 (Figure 1F), resulting in skipping of 46 amino acids NP_056127.2:p.(V825_Q872del).

During our work, Gueneau et al first described Alkuraya-Kucinskas syndrome (MIM 617822), which is caused by homozygous and compound heterozygous variants in the KIAA1109 gene. Clinical manifestations of this syndrome combine severe brain malformations (in particular, ventriculomegaly) and arthrogryposis. Besides that, it includes club foot and heart defects. All these defects were observed in the examined fetus. It allows us to confirm pathogenicity of the identified variants. $^{5}$

One year after, the fourth pregnancy occurred in a spontaneous attempt. It was a dichorionic twin pregnancy, in which fetus II.4.1 did not reveal ultrasonographic abnormalities at 11 to 12 weeks, while fetus II.4.2 presented bilateral ventriculomegaly, increased thickness of the nuchal-fold, arthrogryposis, abnormal fixed feet position, altered profile and atrioventricular canal defect (Figure 1D). Cytogenetic testing of DNA from CVS revealed 46,XY karyotype of both fetuses. Sanger sequencing revealed that fetus II.4.1 had one variant c.1932-3A>G, while fetus II.4.2 had both pathogenic alleles. Based on the analysis, the selective termination of 11.4 .2 was performed at 12 to 13 weeks. The pregnancy with fetus II.4.1 continued normally and resulted in the birth of a healthy baby.

ORCID

Alexandra Filatova (1) https://orcid.org/0000-0002-5979-0727

Ekaterina Lozier (1) https://orcid.org/0000-0003-2901-0539

Alexandra Filatova, ${ }^{1}$ (D) Valeria Freire, ${ }^{1}$ Ekaterina Lozier, ${ }^{2}$ (C) Fedor Konovalov, ${ }^{2}$ Ludmila Bessonova, ${ }^{1}$ Elena ludina, ${ }^{3}$ Valentina Gnetetskaya, ${ }^{3}$ Ilya Kanivets, ${ }^{2}$ Sergey Korostelev, ${ }^{2,4}$ and Mikhail Skoblov, ${ }^{1,5}$

${ }^{1}$ Research Centre for Medical Genetics, Moscow, Russia

${ }^{2}$ Genomed Ltd., Moscow, Russia

${ }^{3}$ MD Medical Group "Mother and Child", Moscow, Russia

${ }^{4}$ Institute of Professional Education, I. M. Sechenov First Moscow State Medical University, Moscow, Russia

${ }^{5}$ School of Biomedicine, Far Eastern Federal University, Vladivostok, Russia

Correspondence

Alexandra Filatova, Research Centre for Medical Genetics, 1 Moskvorechie St., Moscow 115522, Russia.

Email:maacc@yandex.ru

Peer Review

The peer review history for this article is available at https://publons.com/publon/10.1111/cge.13472/

\section{REFERENCES}

1. Fu F, Li R, Li Y, et al. Whole exome sequencing as a diagnostic adjunct to clinical testing in fetuses with structural abnormalities. Ultrasound in 
(A)

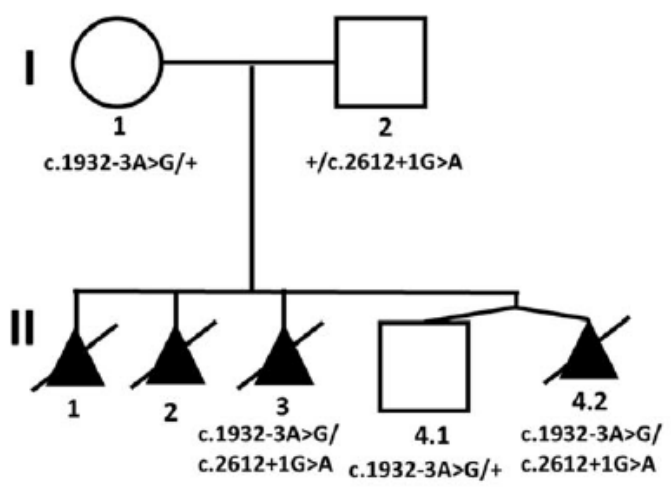

(B)

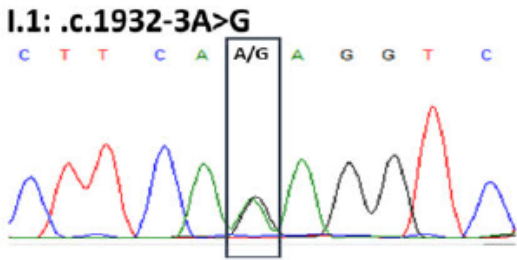

I.2: .c. $2613+1 \mathrm{G}>\mathrm{A}$

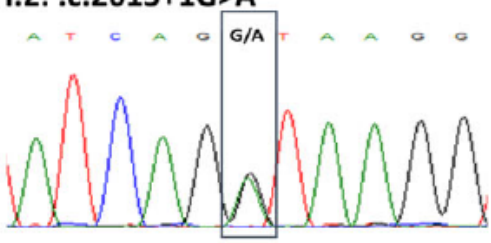

(E)

$$
\text { c.1932-3A>G }
$$

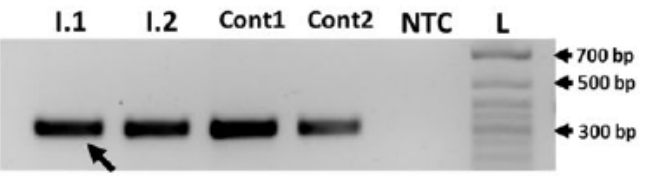

1.
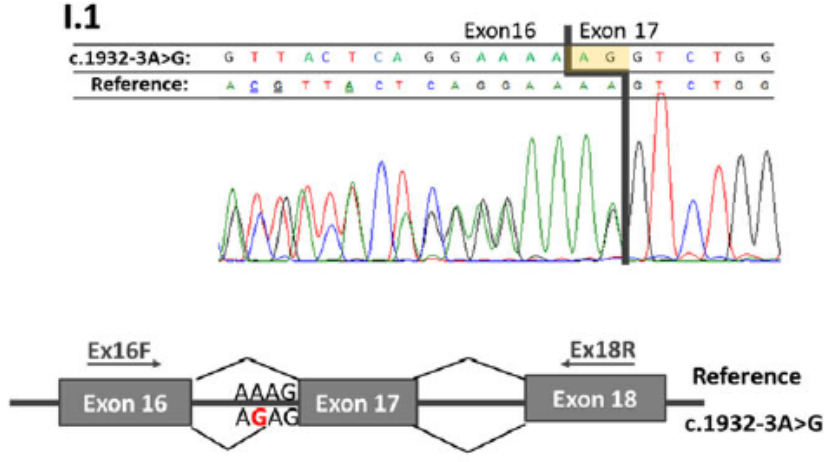

(D) $\quad$ II.4.2
(C) $\mathbf{I I . 3}$
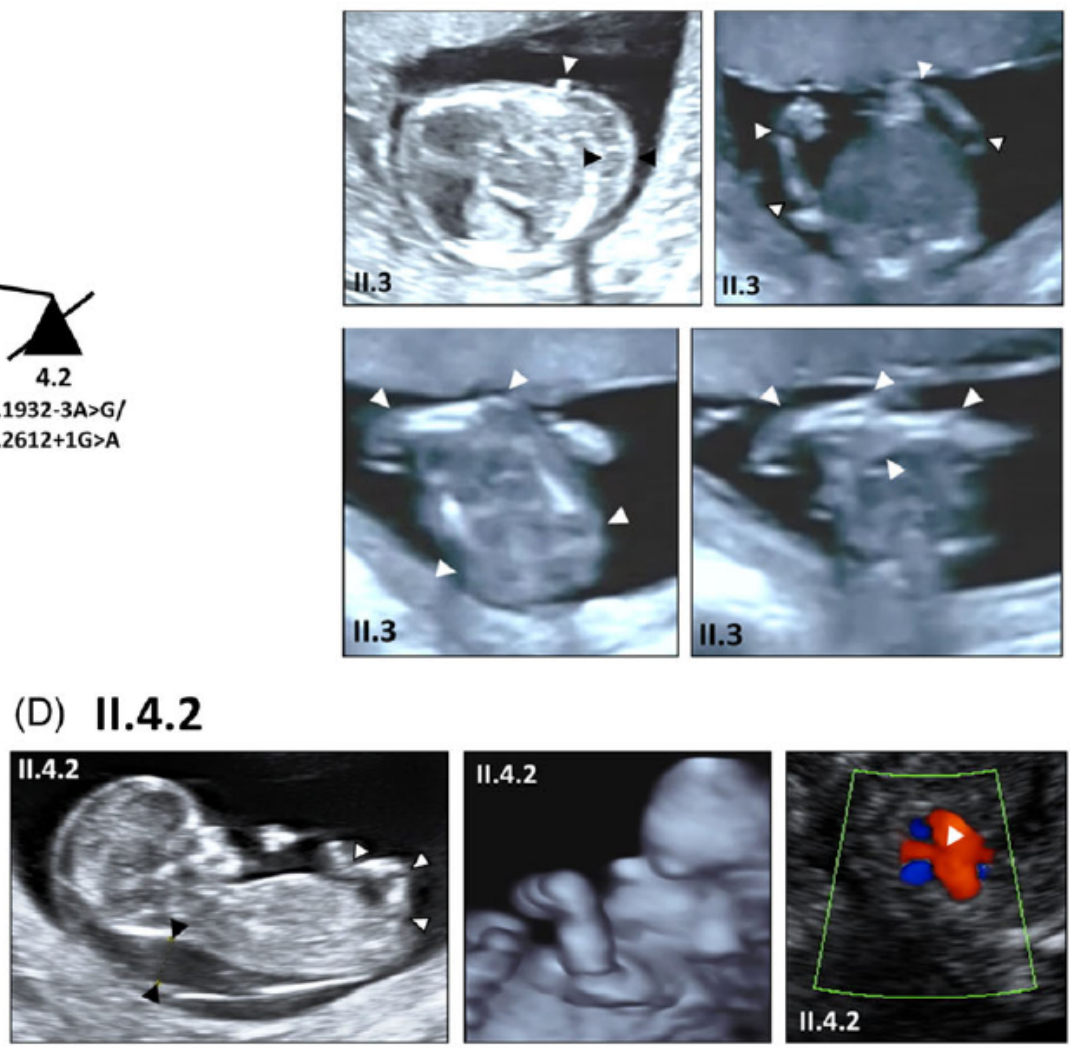

(F) $\quad$ c.2613+1G $>A$

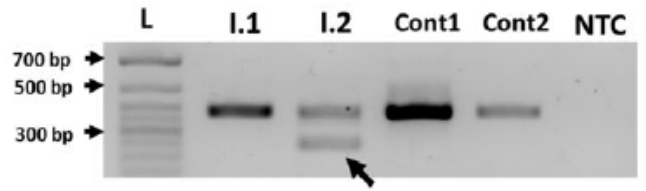

1.2

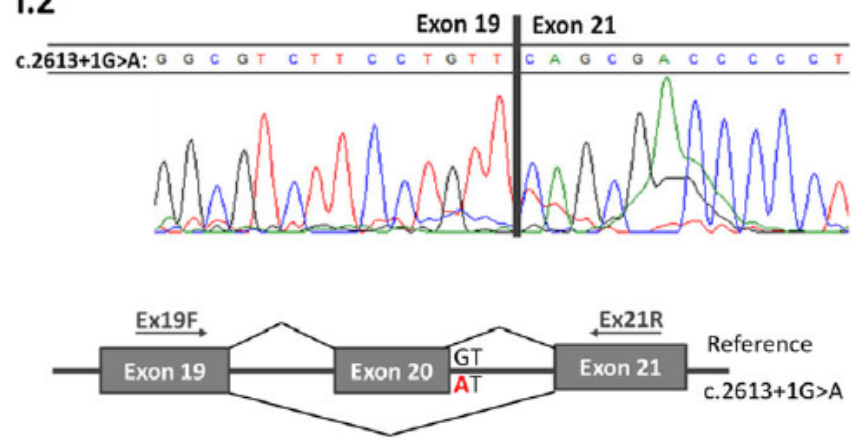

FIGURE 1 Pedigree of the Russian family carrying novel KIAA1109 variants (A). Sanger sequencing results from DNA of the parents (B). Ultrasound scan of affected fetuses: II.3 (C) and II.4.2 (D). RT-PCR analysis of RNA of the parents and two controls for c.1932-3A>G (E) and for c. $2613+1 G>A(F)$ variants. RT-PCR, reverse transcription polymerase chain reaction [Colour figure can be viewed at wileyonlinelibrary.com]

Obstetrics \& Gynecology: The Official Journal of the International Society of Ultrasound in Obstetrics and Gynecology. 2018;51(4):493-502.

2. Dickinson ME, Flenniken AM, Ji X, et al. High-throughput discovery of novel developmental phenotypes. Nature. 2016;537(7621):508-514.

3. Alazami AM, Patel N, Shamseldin HE, et al. Accelerating novel candidate gene discovery in neurogenetic disorders via whole-exome sequencing of prescreened multiplex consanguineous families. Cell Rep. 2015;10(2):148-161.
4. Gueneau L, Fish RJ, Shamseldin HE, et al. KIAA1109 variants are associated with a severe disorder of brain development and arthrogryposis. Am J Hum Genet. 2018;102(1):116-132.

5. Richards S, Aziz N, Bale S, et al. Standards and guidelines for the interpretation of sequence variants: a joint consensus recommendation of the American College of Medical Genetics and Genomics and the Association for Molecular Pathology. Genet Med. 2015;17(5):405-424. 